\title{
ganters
}

\section{Genotype determines weight loss after bariatric surgery}

Genetic factors strongly contribute to the degree of weight loss after Roux-en-Y gastric bypass (RYGB) in individual patients, say US researchers.

The weight loss response after RYGB varies strikingly among different patients. Initially, mechanical factors (variation in surgical technique) or behavioral factors were thought to underlie this variation. However, clinical and behavioral predictors of weight loss can explain only a fraction of the variation in weight loss after surgery. Recent findings that weight loss after RYGB results primarily from surgeryinduced changes in metabolic physiology and signaling raised the question of whether variation in outcome might be due to genetic factors.

Hatoum et al. identified 26 first-degree relatives (siblings or parent-child pairs) living apart who had undergone RYGB and compared their weight loss after surgery to that of 20 patients undergoing RYGB who were living together but were genetically unrelated. "We surmised that, if there were strong genetic contributions to outcome, then people who are closely related should have less difference in their weight loss than those who are unrelated," explains senior investigator Lee Kaplan (Harvard Medical School). Random pairings of the remainder of the cohort, who were not related either genetically or by living arrangements, were used as additional controls.

The average degree of similarity in weight loss after surgery was analyzed within the pairs of patients. The researchers observed that weight loss in genetically related pairs was much more similar than in either group of pairs of genetically unrelated patients. These findings confirm their initial hypothesis that genetic factors contribute to outcomes and support the important role of biological factors in determining the degree of weight loss after RYGB.

Kaplan and his group are currently attempting to identify the specific genes involved in the weight loss response to RYGB. Identification of these genes could

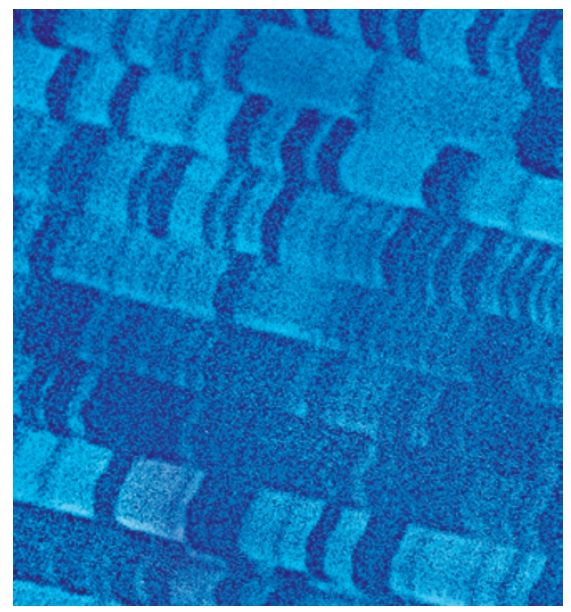

help determine which patients are likely to benefit most from RYGB. Moreover, research on these genes can provide important clues to the physiological mechanisms by which the surgery works, thus facilitating the development of less invasive, nonsurgical approaches that exploit these mechanisms. "We hope to provide prospective patients with a roadmap to the most effective therapy for them as individuals," concludes Kaplan.

\section{Linda Koch}

Original article Hatoum, I. J. et al. Heritability of the weight loss response to gastric bypass surgery. J. Clin. Endocrinol. Metab. doi:10.1210/jc.2011-1130 